\title{
IDS-Pressegespräch
}

\section{Aktionsbündnis gesundes Implantat präsentiert Qualitätssiegel}

Mit dem Label „ImplantatPflegeCenter“ möchte das Aktionsbündnis gesundes Implantat in Zusammenarbeit mit der Deutschen Gesellschaft für Dentalhygieniker/innen DGDH e.V. ein Qualitätssiegel für die Prophylaxe an Implantaten etablieren. Praxen, deren Prophylaxekonzept bei Implantatpatienten auf die Prävention periimplantärer Infektionen ausgerichtet ist und den Anforderungen des Bündnisses entspricht, können so ihre besondere Kompetenz bei der Implantatpflege nach außen demonstrieren. Erstmals vorgestellt wurde das Siegel während des IDSPressegesprächs des Aktionsbündnisses am 11. März 2015 im Rahmen der IDS in Köln.

„Der Schlüssel zur Prävention periimplantärer Infektionen liegt in der regelmäßigen professionellen Implantatreinigung und der nachhaltigen Motivation der Patienten - vor allem durch engagiertes Fachpersonal“, erklärte Jan-Philipp Schmidt, Geschäftsführer des Aktionsbündnisses, während des Pressegesprächs. Die Verleihung des Qualitätssiegels „ImplantatPflegeCenter“ wird auf einem mehrstufigen Verfahren basieren. „Gemeinsam mit unserem wissenschaftlichen Beirat und den dem Aktionsbündnis angeschlossenen Fachverbänden DGDH, BDIZ EDI und GPZ erarbeiten und veröffentlichen wir in den nächsten Monaten geeignete, transparente und patientenrelevante Kriterien, die zum Führen des Labels ,ImplantatPflegeCenter' berechtigen“, kündigte Jan-Philipp Schmidt an.

Praxen, die ihre Implantatprophylaxe als ImplantatPflegeCenter des Aktionsbünd-

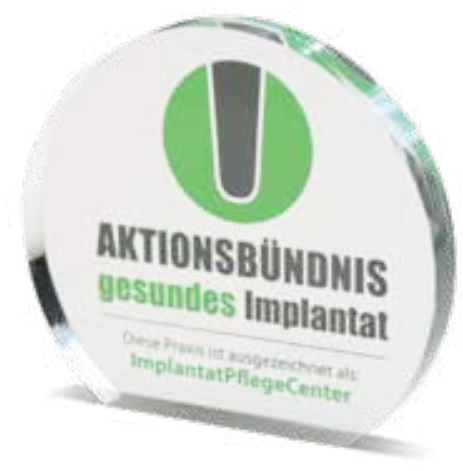

ImplantatPflegeCenter erhalten vom Aktionsbündnis gesundes Implantat diesen hochwertigen Tresenaufsteller aus Plexiglas.

nisses gesundes Implantat evaluieren lassen möchten, können sich ab sofort unter www.gesundes-implantat.de registrieren lassen und erhalten alle notwendigen Informationen und Unterlagen, sobald die Evaluierung startet.

Nach einer Pressemitteilung des Aktionsbündnis gesundes Implantat c/o DentaMedica GmbH, Leipzig 